\title{
Licochalcone A suppresses hexokinase 2-mediated tumor glycolysis in gastric cancer via downregulation of the Akt signaling pathway
}

\author{
JIAN WU ${ }^{1 *}$, XINGXING ZHANG ${ }^{2 *}$, YAOHUI WANG ${ }^{3}$, QINGMIN SUN $^{4}$, \\ MIN CHEN ${ }^{5}$, SHENLIN LIU ${ }^{6}$ and XI ZOU \\ ${ }^{1}$ Central Laboratory, ${ }^{2}$ Digestive Department, ${ }^{3}$ Pathology Department, ${ }^{4}$ Clinical Pharmacy Department, ${ }^{5}$ General Internal \\ Medicine Department, ${ }^{6}$ Oncology Department, The Affiliated Hospital of Nanjing University of Chinese Medicine, \\ Jiangsu Province Hospital of Traditional Chinese Medicine, Nanjing, Jiangsu 210029, P.R. China
}

Received May 20, 2017; Accepted December 5, 2017

DOI: $10.3892 /$ or.2017.6155

\begin{abstract}
Licochalcone A (LicA) is a chalcone extracted from liquorice which has been used as a traditional Chinese medicine for many generations. Increased glucose consumption and glycolytic activity are important hallmarks of cancer cells, and hexokinase 2 (HK2) upregulation is a major contributor to the elevation of glycolysis. Recently, the antitumor activities of LicA have been reported in various cancers; however, its effect on tumor glycolysis in gastric cancer and the underlying mechanisms are completely unknown. In vitro, cell proliferation and clonogenic survival were substantially inhibited after LicA treatment. LicA reduced HK2 expression, and both glucose consumption and lactate production in gastric cancer cells were significantly suppressed. Mechanistic investigations revealed that multiple signaling pathways including Akt, ERK and $\mathrm{NF}-\kappa \mathrm{B}$ were suppressed by LicA. Further studies demonstrated that the inhibition of glycolysis by LicA was mainly attributed to the blockade of the Akt signaling pathway, and the suppression of glycolysis was substantially attenuated when Akt was exogenously overexpressed. In addition to the role in the inhibition of glycolysis, reduction in HK2 was confirmed to be involved in the induction of cell apoptosis. The apoptosis induced by LicA was substantially impaired after HK2 overexpression in gastric cancer cells. The in vivo experiment showed that MKN45 xenograft growth was markedly delayed after LicA treatment and HK2 expression in
\end{abstract}

Correspondence to: Professor Shenlin Liu or Professor Xi Zou, Oncology Department, The Affiliated Hospital of Nanjing University of Chinese Medicine, Jiangsu Province Hospital of Traditional Chinese Medicine, 155 Jianye Road, Nanjing, Jiangsu 210029, P.R. China

E-mail: slliu_bio@126.com

E-mail:xizou_bio@163.com

*Contributed equally

Key words: licochalcone A, tumor glycolysis, apoptosis, hexokinase 2, gastric cancer
LicA-treated tissues was markedly decreased. All of these data demonstrated that blockade of the Akt/HK2 pathway was the underlying mechanism required for LicA to exert its biological activities in glycolysis inhibition and apoptosis induction.

\section{Introduction}

Glucose is the energy source and the important metabolic intermediate in mammalian cells. Under a condition of sufficient oxygen, glucose is metabolized into $\mathrm{H}_{2} \mathrm{O}$ and $\mathrm{CO}_{2}$ via the Krebs cycle to generate abundant ATP to satisfy energy requirements. Different from normal cells, in tumor cells glucose is converted to pyruvate and lactate even in the presence of oxygen, which is termed as aerobic glycolysis or the Warburg effect (1). Increased glucose consumption and glycolytic activity are important hallmarks of cancer cells (2). Although tumor glycolysis is not efficient in terms of generating ATP, it supplies a large number of intermediate products for the synthesis of nucleotides, amino acids and lipids which are required for cell proliferation. Moreover, the accumulation of lactate in tumor tissues results in an acidic microenvironment which enhances chemotherapy resistance, tumor migration and metastasis (3). Owing to the importance of tumor glycolysis, increasing attention has been given on how to interfere with this process of cancer cells to provide a promising therapeutic strategy. The first step of glucose metabolism is the conversion to glucose-6-phosphate (G-6P), which is irreversible and is mainly mediated by hexokinases (HKs). To date, four isoforms of HK (HK1-4) have been identified in mammalian tissue. Among all $\mathrm{HKs}$, hexokinase 2 (HK2) upregulation is a major contributor to the elevation of glycolysis. It has been found that HK2 is abnormally expressed in various types of cancers, for instance gastric (4), breast (5) and colorectal cancer (6) and hepatocellular carcinoma (7), and its expression levels are closely associated with tumor grade, prognosis and mortality $(6,8,9)$.

In contrast with small molecules developed through rational chemical design, natural products have more potential to be applied to tumor chemotherapy owing to their structural diversity. Recently, numerous natural compounds have been demonstrated to exert antitumor effects by inhibiting cell growth, promoting cell death and suppressing 
angiogenesis (10-12). Licochalcone A (LicA) (Fig. 1A) is a characteristic chalcone derived from liquorice, which is also named as 'Gancao' and used as a Traditional Chinese Medicine for many generations. LicA was reported to possess different biological activities, including anti-oxidative (13), anti-inflammatory (14), antiviral and antimicrobial activities (15). Recently, increasing evidence has demonstrated that LicA exhibits profound antitumor activities in various types of cancers, such as breast (16), non-small cell lung (17) and cervical cancer. Induction of cell apoptosis, cell cycle arrest and autophagy $(16,18,19)$, inhibition of migration and metastasis (20), and blockade of angiogenesis in tumor tissues (21) have been identified to be the underlying mechanisms.

Although antitumor activities of LicA have been verified, its effect on tumor glycolysis remains largely unknown. In the present study, we investigated the effect of LicA on tumor glycolysis in gastric cancer cells as well as the underlying mechanisms. The results demonstrated that LicA exhibited substantial activities against tumor glycolysis by reducing HK2 expression. Further investigation illustrated that the decrease in HK2 by LicA was due to the blockade of the Akt signaling pathway.

\section{Materials and methods}

Cell lines and reagents. Gastric cancer cell lines MKN45 and SGC7901 and the gastric epithelial cell GES-1 were purchased from the Chinese Committee of Type Culture Collection Cell Bank, Chinese Academy of Sciences (Shanghai, China). MKN45 and SGC7901 cells were cultured in Dulbecco's modified Eagle's medium (DMEM) containing 10\% FBS and $1 \%$ antibiotics. The GES- 1 cells were cultured in RPMI-1640 medium containing $10 \%$ FBS and $1 \%$ antibiotics. LicA and specific signaling pathway inhibitors including LY294002, PD98059, Bay11-7082 and parthenolide were purchased from Selleck (Shanghai, China). Primary anti-HK2 (\#2867), Glut1 (\#12939), PKM2 (\#4053), LDHA (\#3582), p-Akt

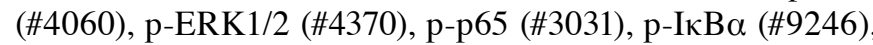
p-S6 (\#4858), p-GSK3ß (\#12456), Akt1 (\#75692), MCL-1 (\#5453), BCL2 (\#2870), BCL-XL (\#2764), cleaved caspase-3 (\#9664), cleaved PARP (\#5625) antibodies and secondary antibodies anti-rabbit IgG HRP (\#7074) and anti-mouse IgG HRP (\#7076) were products of Cell Signaling Technology Inc. (Beverly, MA, USA). Anti- $\beta$-actin antibody (A5316) was purchased from Sigma-Aldrich (St. Louis, MO, USA). Myr-Akt1 plasmid was purchased from Addgene (Cambridge, MA, USA). HK2 (ORF004940) construct was a product of Applied Biological Materials (ABM) Inc. (Richmond, BC, Canada). Lipofectamine ${ }^{\circledR} 2000$ was purchased from Invitrogen (Carlsbad, CA, USA). Annexin V-FITC and propidium iodide were products of Biolegend (San Diego, CA, USA).

Cell proliferation assay. MKN45 or SGC7901 cells in logarithmic growth phase $\left(3 \times 10^{3} /\right.$ well) were seeded into 96 -well plate. Twenty-four hours later, the cells were treated with various concentrations of LicA. At different time points (24, 48 or 72 h), CellTiter96 Aqueous One Solution (Promega Corp., Madison, WI, USA) (20 $\mu \mathrm{l} /$ well) was added to a 96-well plate and the cell viability was assessed according to the manufacturer's protocol.
Clonogenic survival assay. Cells cultured in 10-cm plastic dishes were treated with different concentrations of LicA for $24 \mathrm{~h}$, and the cells were harvested and reseeded into a 6-well plate in duplicate at the appropriate density. The cells were cultured for 1-3 weeks until the colonies with substantially good size (minimum 50 cells per colony) were formed in the control group. After washing with PBS, the colonies were fixed with the fixation solution (methanol:acetic acid 3:1) and then stained with $0.5 \%$ crystal violet for $2 \mathrm{~h}$ at room temperature. The crystal violet was carefully removed by immersing the plates in tap water repeatedly and the plates were air-dried. The number of colonies was photographed and counted.

Western blot analysis. After the treatment of LicA, gastric cancer cells were lysed with RIPA lysis buffer containing protease cocktail (Roche, Mannheim, Germany) on ice for $30 \mathrm{~min}$. The cell lysate was harvested and centrifugated at $12,000 \mathrm{x} g$ for $5 \mathrm{~min}$, and the supernatant was collected. The protein concentrations were determined by the Bradford assay (Bio-Rad, Philadelphia, PA, USA). Twenty micrograms per sample was subjected to SDS-PAGE and then transferred onto polyvinylidene difluoride membranes (Millipore, Billerica, MA, USA). After blocking the non-specific binding site on the membrane with $5 \%$ non-fat milk solution, the membranes were incubated with specific primary antibodies at a dilution of $1: 1,000$ at $4^{\circ} \mathrm{C}$ overnight. After washing three times with TBS-Tween-20, the membranes were incubated with HRP-conjugated secondary antibody at a dilution of 1:2,000 at room temperature for $1 \mathrm{~h}$, and then the bands on the membrane were visualized using an enhanced chemiluminescence reagent (Thermo Fisher Scientific, Inc., Waltham, MA, USA).

Glucose uptake and lactate production measurement. Gastric cancer cells $\left(5 \times 10^{5} /\right.$ well) were plated in 6-well tissue culture plate and cultured overnight. Then, the culture medium was discarded and cells were incubated with fresh medium containing different concentrations of LicA for $12 \mathrm{~h}$. The Automatic Biochemical Analyzer (7170A, Hitachi, Tokyo, Japan) was used for the measurement of the levels of glucose and lactate in the culture medium. Protein concentration per sample acted as the control to normalize the relative glucose consumption and lactate production rate.

Flow cytometry. After LicA treatment, MKN45 cells and the cell culture medium were collected and centrifugated. After washing with PBS, the cell pellets were resuspended and stained with Annexin V-FITC and propidium iodide according to the manufacturer's instructions, and then the stained cells were subjected to flow cytometry. The results were analyzed and quantified by a flow cytometer (BD Biosciences, San Jose, CA, USA).

Cell transfection. For Myr-Akt1 or pORF-HK2 transfection, HCC cells were seeded into a 6-well culture plate and grown to $70-90 \%$ confluence. Before transfection, the culture medium was replaced with $1.5 \mathrm{ml}$ fresh medium without fetal bovine serum. Plasmid DNA $(2.5 \mu \mathrm{g})$ and Lipofectamine 2000 reagent $(7.5 \mu \mathrm{l})$ were diluted with $150 \mu \mathrm{l}$ Opti-MEM medium respectively and incubated at room temperature for $5 \mathrm{~min}$. The diluted DNA and transfection reagent were mixed and incubated for 10 mins avoiding light, then $250 \mu \mathrm{l}$ mixture 
A<smiles>C=CC(C)(C)c1cc(O)cc(/C=C/C(=O)c2ccc(O)cc2)c1</smiles>

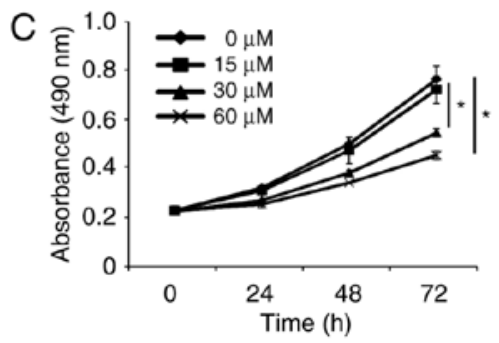

D
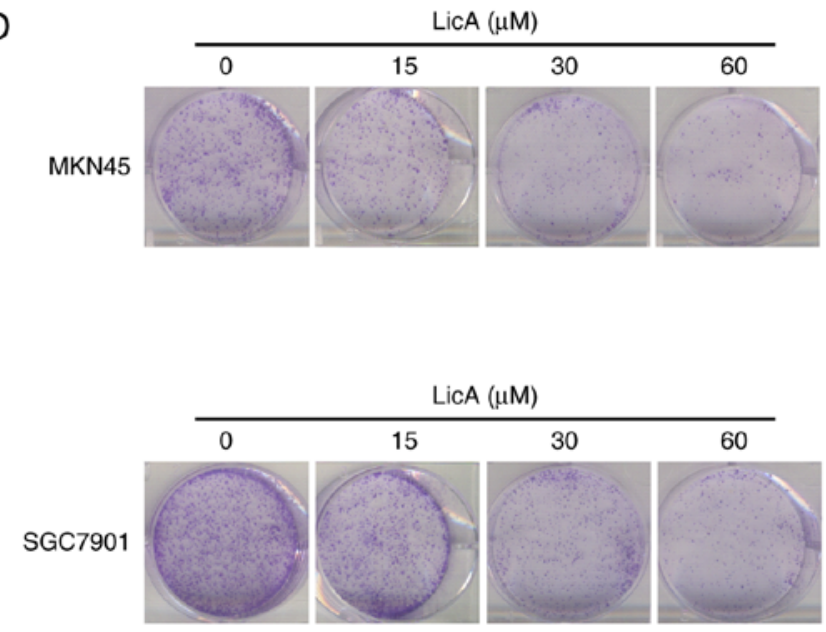

B
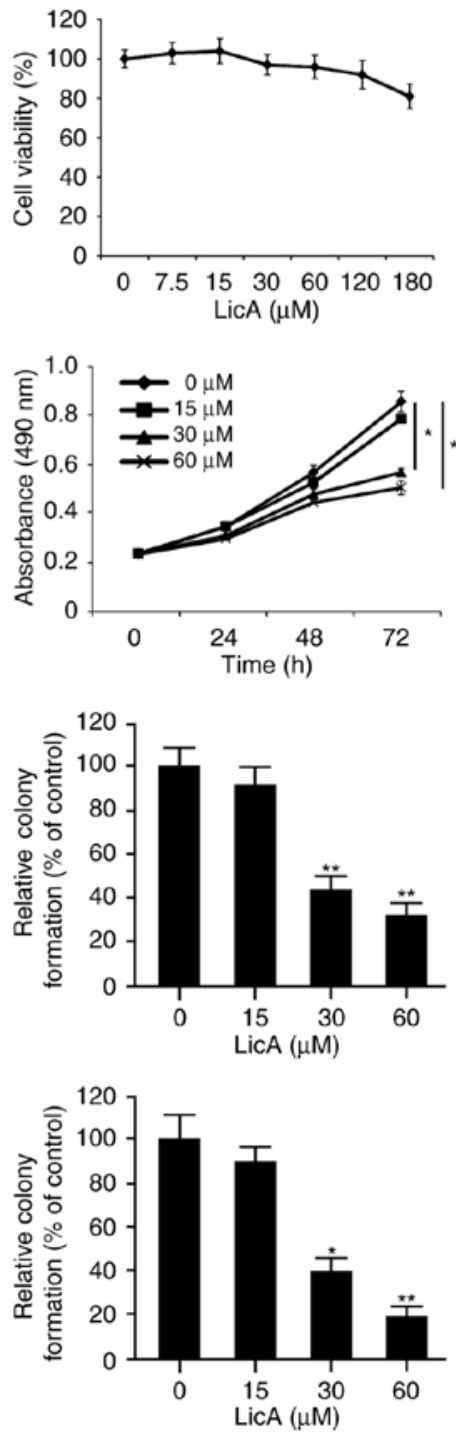

Figure 1. LicA inhibits gastric cancer cell proliferation and clonogenic survival in vitro. (A) Chemical structure of LicA. (B) The effect of LicA on normal gastric epithelium GES-1 cells. The GES-1 cells were treated with the indicated concentrations of LicA for $72 \mathrm{~h}$, and the cytotoxicity was determined by MTS assay. (C) LicA inhibited gastric cancer cell proliferation. MKN45 (left) and SGC7901 cells (right) were incubated with the indicated concentrations of LicA for different times and the cell proliferation was examined by the MTS assay. " $\mathrm{P}<0.05$ indicates a significant decrease in cell proliferation. (D) LicA suppressed the clonogenic survival of gastric cancer cells. Clonogenic survival assays in MKN45 (upper) and SGC7901 (lower) cells were performed as described in Materials and methods. Representative images are shown (left panels). The results of three independent experiments are expressed as mean \pm SD (right panels), ${ }^{*} \mathrm{P}<0.05 ;{ }^{* * *} \mathrm{P}<0.01$ (Student's t-test) indicate a significant difference vs. the control. LicA, licochalcone.

was added per well and the culture medium was replaced with fresh medium with fetal bovine serum after 4-6 h. Forty-eight hours later, the transfected cells were used for further studies.

Xenograft mouse model. In accordance with the protocol approved by the Institutional Animal Care and Use Committee, BALB/ca nude mice maintained under specific pathogen-free (SPF) conditions were subcutaneously injected with MKN45 cells $\left(2 \times 10^{6}\right.$ cells/mice). Once the tumor was formed and the volume was $\sim 50 \mathrm{~mm}^{3}$, the mice were randomly assigned into a vehicle and experimental group. The vehicle group received $0.2 \mathrm{ml}$ sterile PBS solution, and the experimental group was treated with $10 \mathrm{mg} / \mathrm{kg}$ LicA by i.p. injection daily. The tumor volume (V) was measured twice per week with microcalipers and was calculated as $V=\left(\right.$ length $\mathrm{x}$ width $\left.^{2}\right) / 2$. At the end of the experiment, the mice were sacrificed and the tumors were removed.
Immunohistochemical staining. Tumor tissues isolated from the mice were embedded in paraffin and cut into 5- $\mu \mathrm{m}$ sections. After dewaxing in xylene and hydration in serial ethanol, the slides were boiled in sodium citrate buffer $(10 \mathrm{mmol} / \mathrm{l}, \mathrm{pH}$ 6.0) for $10 \mathrm{~min}$ to expose antigens. To block endogenous peroxidase, the slides were treated with $3 \% \mathrm{H}_{2} \mathrm{O}_{2}$ for $10 \mathrm{~min}$. After incubation with goat serum at room temperature for $1 \mathrm{~h}$, the slides were incubated with the anti-HK2 (1:100) or anti-Ki-67 (1:200) antibody respectively at $4^{\circ} \mathrm{C}$ in a humidified chamber overnight. Following washing with PBS three times, the slides were hybridized with biotinylated goat anti-rabbit secondary antibody at room temperature for $30 \mathrm{~min}$. After washing with PBS, the sections were probed with HRP-conjugated streptavidin and then visualized with DAB solution. After counterstaining with Harris' hematoxylin, the slides were dehydrated and mounted. The intensity was analyzed by Image-Pro PLUS (v.6) and ImageJ (NIH) software programs. 

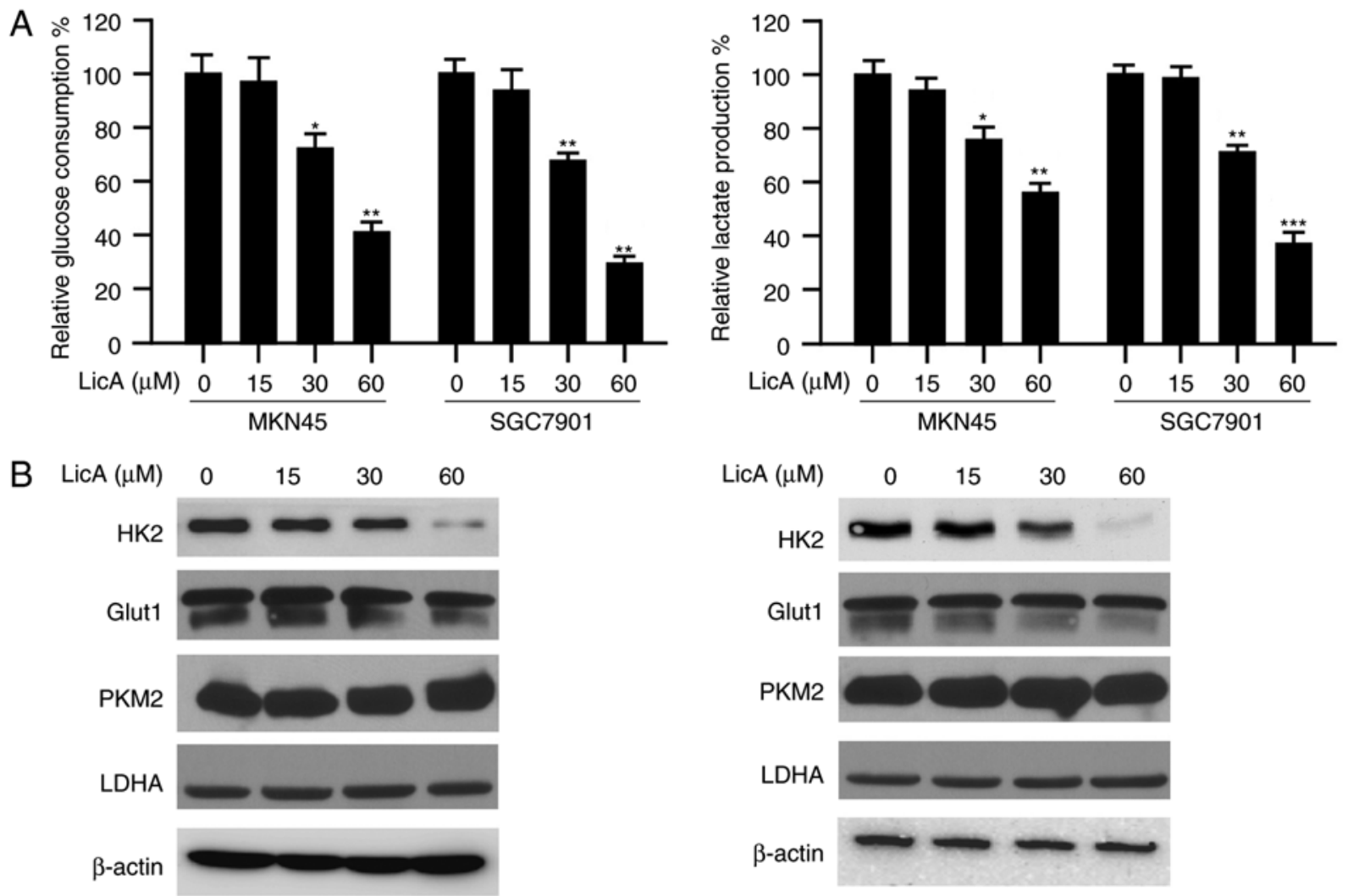

Figure 2. LicA suppresses gastric cancer cell tumor glycolysis via reducing HK2 expression. (A) Glucose consumption (left panels) and lactate production (right panels) in cell culture medium were analyzed as described. The results of three independent experiments expressed as mean $\pm \mathrm{SD}$ are shown, ${ }^{*} \mathrm{P}<0.05$ ${ }^{* *} \mathrm{P}<0.01 ;{ }^{* * *} \mathrm{P}<0.001$ (Student's t-test) indicate a significant difference vs. the control. (B) The expression of key glycolytic enzymes after treatment with LicA MKN45 (left) and SGC7901 (right) cells were treated with various concentrations of LicA and the change in the indicated proteins was examined by western blotting. LicA, licochalcone. HK2, hexokinase 2.

Statistical analysis. SPSS software (version 13.0; IBM SPSS, Armonk, NY, USA) was used for statistical analysis. Student's t-test or one-way ANOVA was adopted to evaluate statistical significance. $\mathrm{P}<0.05$ indicated a statistically significant difference.

\section{Results}

LicA suppresses gastric cancer cell proliferation in vitro. Firstly, we tested the effect of LicA on normal gastric epithelium cell line GES-1. The results showed that LicA had no obvious cytotoxicity at concentrations $\leq 180 \mu \mathrm{M}$ (Fig. 1B). We next examined the anti-proliferative activity of LicA in gastric cancer cells in vitro. As shown in Fig. 1C, at the low concentration $(15 \mu \mathrm{M})$, no obvious inhibitory effect was observed. With the increase in concentration (30-60 $\mu \mathrm{M})$ and the extension of treatment time (48-72 h), cell proliferation in the MKN45 and SGC7901 cells was significantly inhibited. The $\mathrm{IC}_{50}$ of LicA in MKN45 and SGC7901 cells was 63.57 and $55.56 \mu \mathrm{M}$ respectively (data not shown). Beyond that, the effect of LicA on clonogenic survival was also investigated. The incubation of LicA resulted in the decrease in colonies formed in the agar, and the number of clones was substantially decreased in a dose-dependent manner. At $60 \mu \mathrm{M}$, little colonies were formed after exposure to LicA (Fig. 1D). All these data demonstrated that LicA exerted a profound antitumor activity in gastric cancer cells in vitro.
LicA inhibits tumor glycolysis in gastric cancer cells via reducing $H K 2$. In order to study the effect of LicA on tumor glycolysis, the amount of glucose consumption in gastric cancer cells was determined. As shown in Fig. 2A, after LicA treatment, the glucose consumed by MKN45 and SGC7901 cells was significantly decreased dose-dependently. At the high concentration of $60 \mu \mathrm{M}, \sim 50 \%$ inhibition was observed. Accompanied by the reduction of glucose absorption, the amount of lactate secreted by gastric cancer cells was also decreased significantly. Furthermore, we tested the effect of LicA on key glycolytic enzymes and the results demonstrated that the expression of HK2 was decreased by LicA. The expression of other glycolytic enzymes, such as GLUT1, PKM2 and LDHA, had no obvious change (Fig. 2B). All these data imply that HK2 is involved in the suppression of glycolysis mediated by LicA.

Akt signaling pathway is involved in the regulation of HK2 expression by LicA. To confirm which signaling pathway is engaged in the regulation of HK2 expression, the effect of LicA on the main signaling pathways in gastric cancer cells was investigated. As demonstrated, several signaling pathways in gastric cancer, such as ERK, Akt and NF- $\mathrm{KB}$ were blocked by LicA dose-dependently (Fig. 3A). To further validate the inhibition of these pathways, we examined the effect of LicA on EGF-induced activation of ERK and Akt, as well as TNF- $\alpha$-induced NF- $\kappa$ B activation. As shown in Fig. 3B and $\mathrm{C}$, the activation of ERK, Akt and NF- $\mathrm{KB}$ signaling was 

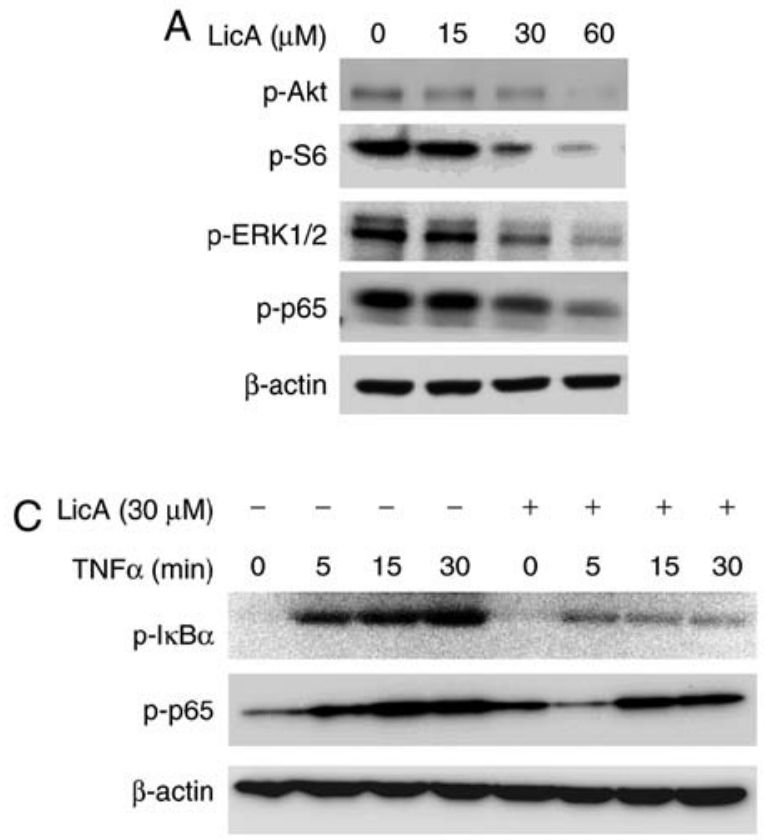

B
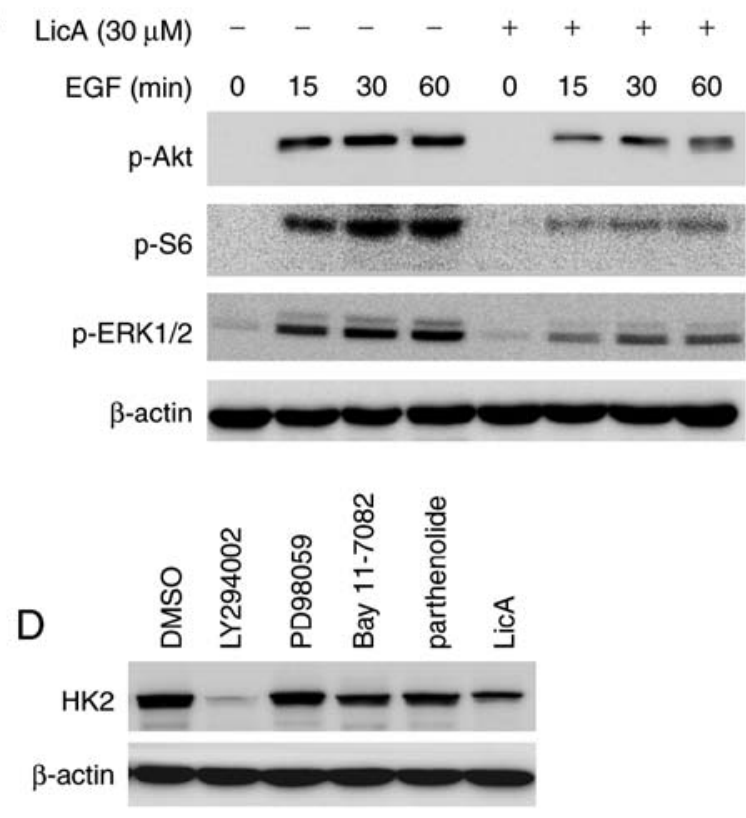

Figure 3. LicA decreases HK2 expression by blocking the Akt signaling pathway. (A) LicA inhibited the signaling pathways in gastric cancer cells. MKN45 cells were incubated with different concentrations of LicA for $24 \mathrm{~h}$ and the effect on phosphorylation of the indicated proteins was examined. (B and C) LicA blocked EGF-induced activation of ERK, Akt and S6 (B), and TNF- $\alpha$-induced activation of the NF- $\mathrm{B}$ signaling pathway (C). MKN45 cells were starved overnight and then treated with $30 \mu \mathrm{M}$ LicA for $2 \mathrm{~h}$. After stimulation with $100 \mathrm{ng} / \mathrm{ml}$ EGF or $30 \mathrm{ng} / \mathrm{ml} \mathrm{TNF}-\alpha$ for the indicated times, cell lysates were collected and western blotting was used to examine the indicated protein. (D) MKN45 cells were treated with specific PI3-K inhibitor (LY294002), MEK inhibitor (PD98059), NF-кB inhibitor (Bay11-7082) and parthenolide, respectively, for $12 \mathrm{~h}$, and cell lysates were probed with anti-HK2 antibodies. LicA, licochalcone. HK2, hexokinase 2.

suppressed by LicA dose and time-dependently. By using different specific signaling pathway inhibitors to treat gastric cancer cells and observe the change in HK2 expression, we identified that LY294002, a specific PI3-K inhibitor, demonstrated a similar effect on HK2 as LicA, implying that the Akt signaling pathway may be involved in the mediation of HK2 expression (Fig. 3D).

Hyperactivation of Akt in gastric cancer cells attenuates LicAmediated glycolysis suppression. Based on the results shown above, the HK2 decrease caused by LicA may be attributed to the blockade of Akt activation, thus, we tested the effect of LicA on the Akt signaling pathway. After LicA treatment, along with the inactivation of Akt, the phosphorylation of S6 and GSK3 $\beta$, which are the main downstream signaling of Akt, were inhibited dose-dependently (Fig. 4A and B). To illustrate the importance of Akt in the regulation of HK2 expression, constitutively activated Akt1 (myr-Akt1) was transfected into MKN45 and SGC7901 cells. As expected, with the recovery of Akt activity in gastric cancer cells, HK2 reduction caused by LicA was notably reversed (Fig. 4C and D). Moreover, glucose consumption and lactate production in myr-Aktl transfected cells were also significantly increased (Fig. 4E and F). These results demonstrated that Akt-mediated HK2 expression plays a pivotal role in the effectd of LicA on tumor glycolysis.

Overexpression of HK2 impairs LicA-induced cell apoptosis. Except glycolysis suppression, LicA also induced apoptosis in gastric cancer cells. The expression of cleaved-caspase-3 and PARP, which are signals of cells undergoing apoptosis, were significantly increased (Fig. 5A and B). The results of
FACS analysis also confirmed that MKN45 cells exhibited induced apoptosis dose-dependently; $25 \%$ of tumor cells were subjected to apoptosis at $60 \mu \mathrm{M}$ (Fig. 5C). Furthermore, the anti-apoptotic proteins such as Bcl-2, and Mcl-1 were found to be decreased after LicA treatment, whereas the expression of pro-apoptotic proteins including Bad and Bak had no significant change. In addition to mediating tumor glycolysis, HK2 is also involved in apoptosis regulation. Given the decrease in HK2 expression by LicA, we speculated that HK2 was also involved in LicA-induced cell apoptosis. After exogenous HK2 overexpression in gastric cancer, the apoptosis induced by LicA was substantially attenuated and the expression of cleaved-PARP and caspase-3 was significantly decreased in contrast with the control group, suggesting that HK2 was involved in LicA-induced apoptosis (Fig. 5D-F).

LicA restrains tumor growth in a gastric xenograft model. The in vivo antitumor activity of LicA was investigated in an MKN45 xenograft model. In contrast with the vehicle group, tumor growth in the LicA-treated group was significantly inhibited (Fig. 6A-C). The tumor volume of the vehicle group had reached about $900 \mathrm{~mm}^{3}$, whereas the average volume of the LicA-treated group was $\sim 400 \mathrm{~mm}^{3}$. The tumor weight of the LicA-treated group was also significantly smaller than the vehicle group ( 0.52 vs. $0.85 \mathrm{~g}$ ). Based on the change in body weight, no obvious toxicity was observed (Fig. 6D). As shown in Fig. 6E, immunohistochemistry staining of LicA-treated tumor tissues demonstrated that the expression of HK2 was substantially decreased. Meanwhile, the expression of $\mathrm{Ki}-67$, a marker of cell proliferative potential, was also decreased, indicating that LicA inhibited tumor growth in vivo by suppressing tumor glycolysis. 

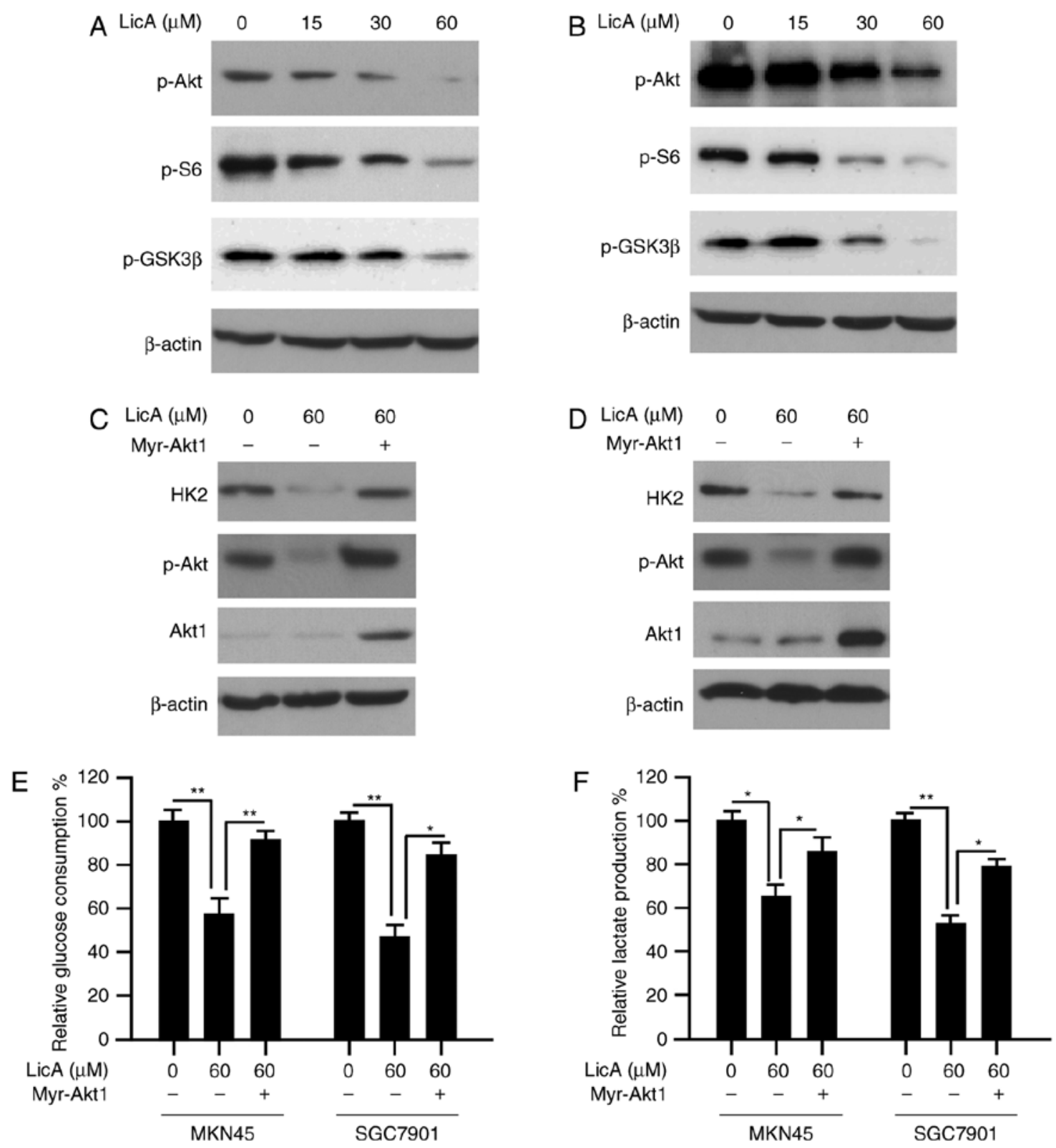

Figure 4. Hyperactivation of Akt attenuates LicA-mediated glycolysis suppression. (A and B) LicA inhibited the Akt signaling pathway and HK2 expression in MKN45 (A) and SGC7901 (B) cells. MKN45 or SGC7901 cells were incubated with LicA for $12 \mathrm{~h}$ and cell lysates were detected with the indicated antibodies. (C and D) Hyperactivation of Akt reversed HK2 suppression by LicA. MKN45 (C) and SGC7901 (D) cells were transfected with Myr-Akt1 plasmid and then treated with $60 \mu \mathrm{M} \mathrm{LicA}$, and western blotting was performed to probe HK2 expression. (E and F) Hyperactivation of Akt impaired glycolysis suppression by LicA. MKN45 and SGC7901 cells were transfected with Myr-Akt1 and then exposed to $60 \mu \mathrm{M} \mathrm{LicA}$. Glucose consumption (E) and lactate production (F) were examined at $12 \mathrm{~h}$ as described. The results of three independent experiments expressed as means $\pm \mathrm{SD}$ are shown, ${ }^{*} \mathrm{P}<0.05$; ${ }^{* *} \mathrm{P}<0.01$ (Student's t-test) indicates significant difference between the different groups. LicA, licochalcone. HK2, hexokinase 2.

\section{Discussion}

Gastric cancer ranks fourth as the most most common malignancy and the second leading cause of cancer-associated mortality. In China, it was estimated the mortality reached $\sim 498,000$ in 2015 (22). Despite the advance in targeted therapy and early diagnosis of gastric cancer, the progress in clinical therapy remains limited. Apart from small molecules synthesized by medical chemistry, natural products have drawn increasing attention in cancer chemopreventive therapies (23). The results of the present study demonstrated that LicA had profound potency against gastric cancer in vitro and in vivo by suppressing tumor glycolysis. Through reducing HK2 expression, the glycolysis in gastric cancer cells was markedly inhibited by LicA. Meanwhile, owing to the decrease in HK2, gastric cancer cells were subjected to induction of apoptosis. Further studies identified that the inhibition of the Akt signaling pathway was involved in the regulation of HK2 activity.

The potency of LicA in different tumor types is highly different. As reported, breast cancer cells demonstrated high sensitivity to LicA with an $\mathrm{IC}_{50}$ of $20 \mu \mathrm{M}(16)$, and the $\mathrm{IC}_{50}$ of LicA in cervical cancer and head and neck cancer was 40 and $100 \mu \mathrm{M}$, respectively $(18,19)$. In our study, the $\mathrm{IC}_{50}$ of LicA in gastric cancer was $\sim 60 \mu \mathrm{M}$, which was in accordance with the results reported by Hao et al (24). In cervical cancer, 


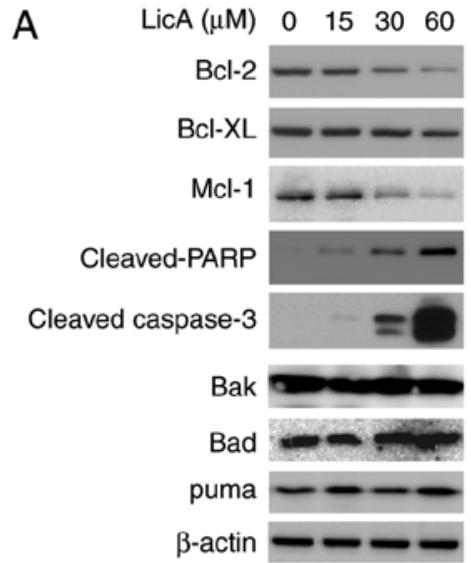

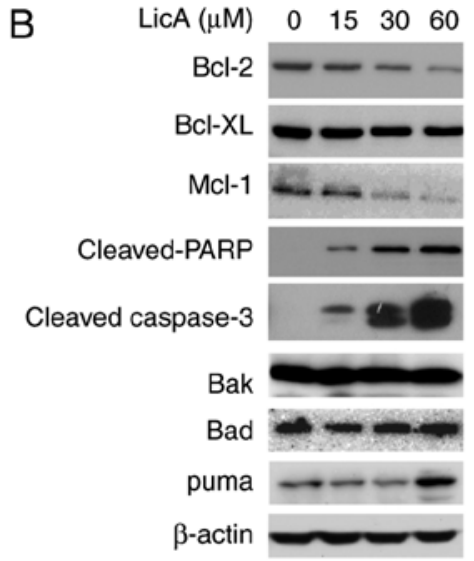
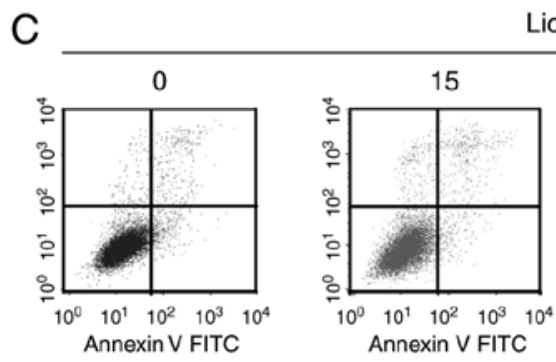

$\operatorname{LicA}(\mu \mathrm{M})$
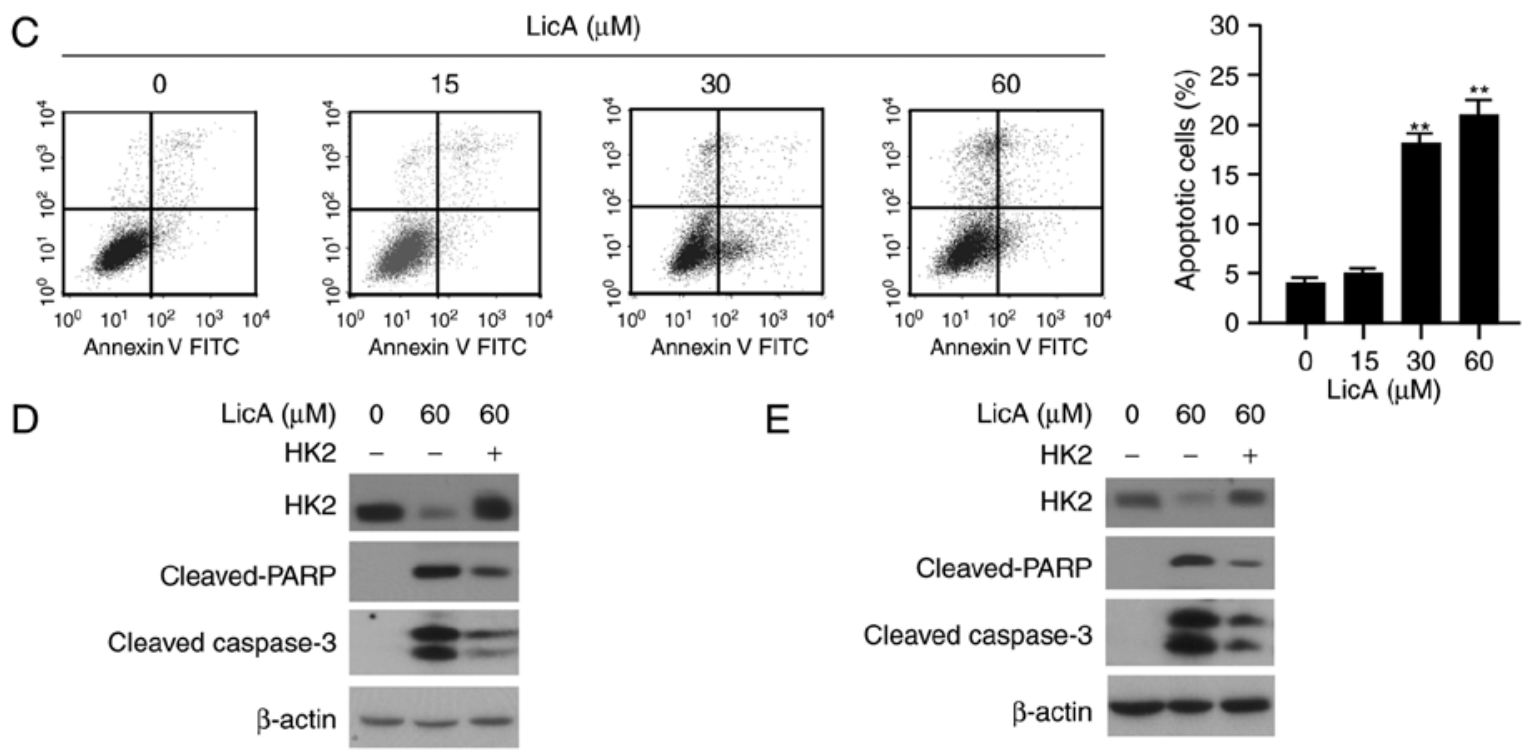

$\mathrm{E}$

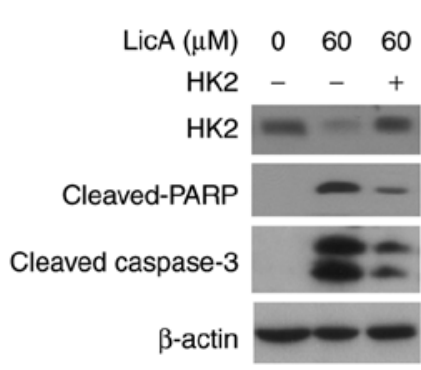

$\mathrm{F}$

$\operatorname{LicA}(\mu \mathrm{M})$
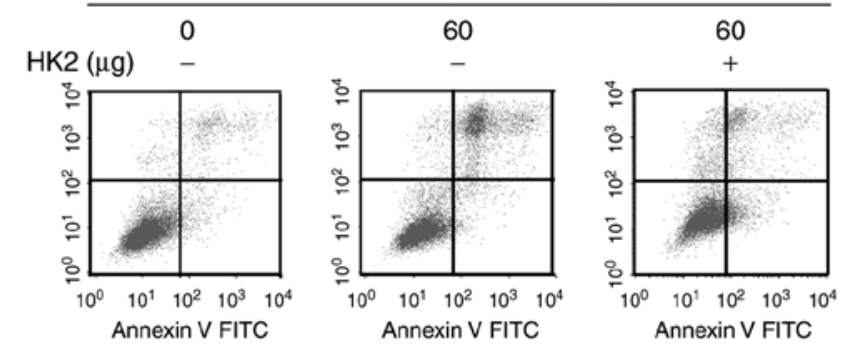

Figure 5. Overexpression of HK2 impairs LicA-induced cell apoptosis. MKN45 (A) and SGC7901 (B) underwent apoptosis after LicA treatment. MKN45 or SGC7901 cells were treated with LicA for $24 \mathrm{~h}$, and cell lysates were collected and examined by western blotting with the indicated antibodies. (C) MKN45 cells were treated with LicA for $24 \mathrm{~h}$ and collected for Annexin V-PI double staining, and then subjected to FACS analysis. (D-F) Overexpression of HK2 decreased cell apoptosis induced by LicA. MKN45 (D) and SGC7901 (E) cells were transfected with pORF-HK2 and then treated with LicA, and cell lysates were examined with the indicated antibodies. (F) MKN45 cells were transfected with pORF-HK2 and then treated with LicA. Then, the cells were collected and stained with Annexin V and PI, and analyzed with FACS. ${ }^{*} \mathrm{P}<0.05,{ }^{* * *} \mathrm{p}<0.01$ and ${ }^{* * *} \mathrm{p}<0.001$ (Student's t-test) indicates significant difference. HK2, hexokinase 2. LicA, licochalcone.

an i.p injection of $10 \mathrm{mg} / \mathrm{kg} \mathrm{LicA}$ substantially inhibited the xenograft growth (19). Although the sensitivity of head and neck cancer to LicA was relatively low, intravenous injection of $10 \mathrm{mg} / \mathrm{kg}$ LicA also significantly delayed the tumor growth (18). Therefore, in our study, we also chose $10 \mathrm{mg} / \mathrm{kg}$ for the in vivo experiments. As expected, the growth of MKN45 xenografts was substantially attenuated by daily treatment of $10 \mathrm{mg} / \mathrm{kg} \mathrm{LicA}$.
Tumor glycolysis is an important hallmark of cancer cells. Clinical observations verified the ${ }^{18} \mathrm{~F}$-FDG uptake is an independent and significant prognostic indicator of tumor recurrence in gastric cancer $(25,26)$. The level of glucose uptake was closely correlated with the overexpression of glycolytic enzymes, such as GLUT, HK2, PKM2, which were found to be overexpressed in gastric cancer tissue and were significantly correlated with disease progression 
A

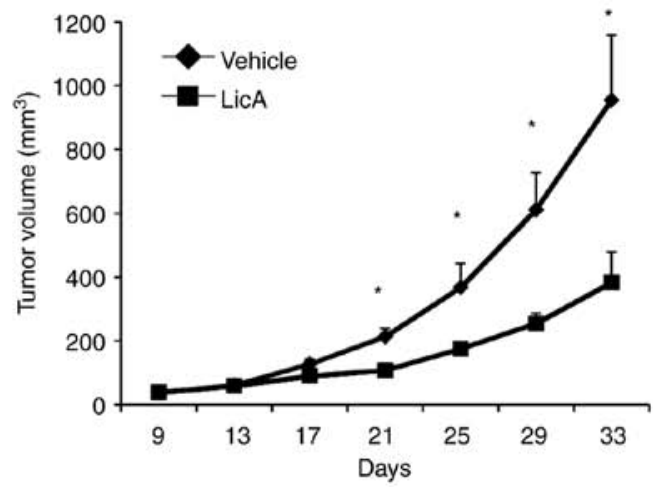

C vehicle LicA

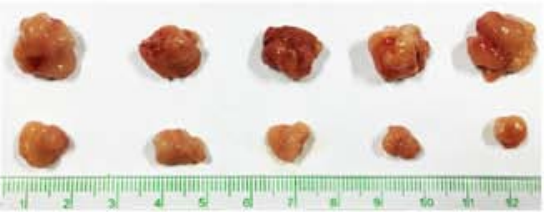

B

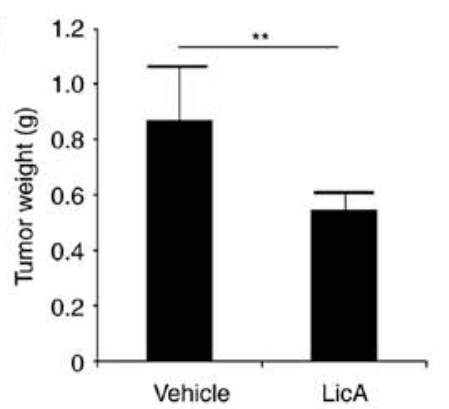

D

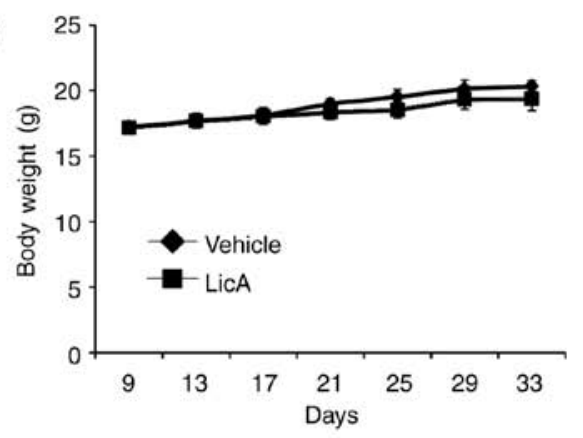

$E$
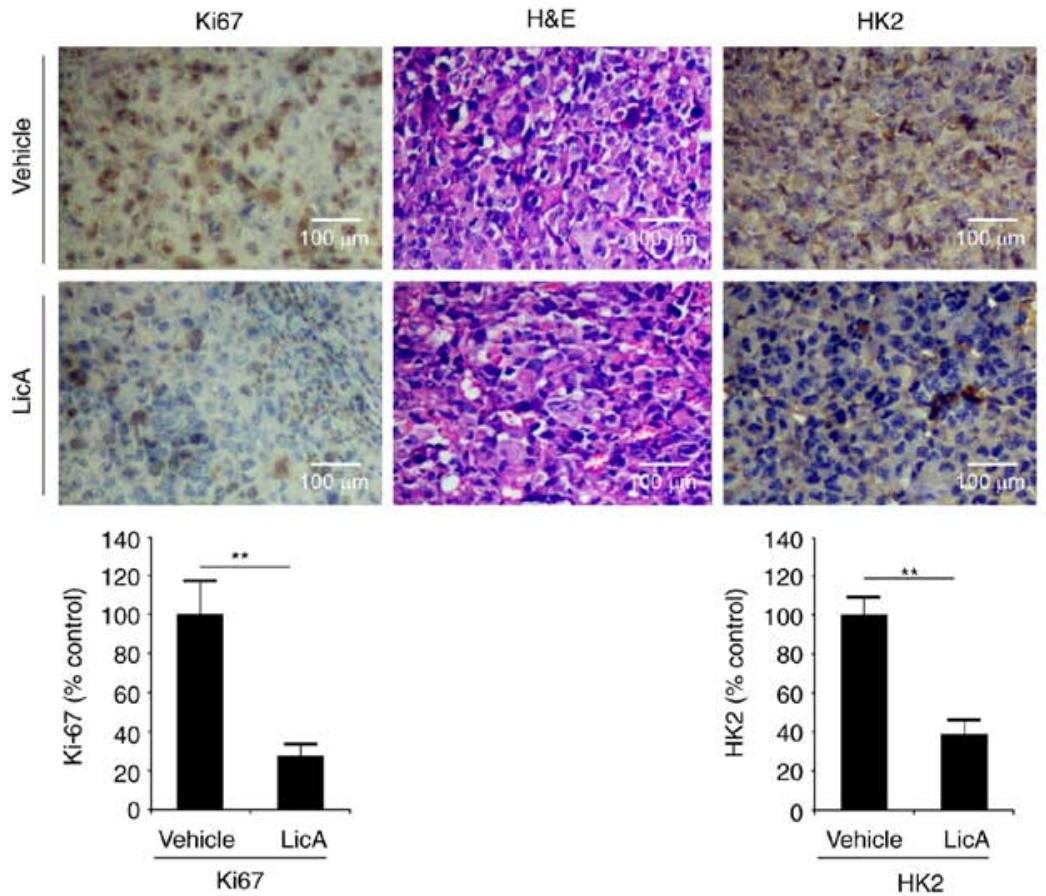

Figure 6. LicA inhibits the growth of an MKN45 xenograft model in vivo. (A) The growth curve of the vehicle and LicA-treated group. (B) The weight of the vehicle and LicA-treated xenografts. (C) Images of the xenografts in the vehicle and treatment group. (D) The body weight curve of tumor-bearing mice. Nude mice with MKN45 xenografts were randomly grouped and $10 \mathrm{mg} / \mathrm{kg} \mathrm{LicA}$ was administered daily by i.p. injection. The tumor volume and body weight were measured twice weekly. (E) Tumor tissues of the vehicle and LicA-treated group were subjected to immunohistochemical staining to examine the expression of $\mathrm{HK} 2$ and $\mathrm{Ki}-67$, respectively. Upper panel, representative images of $\mathrm{HK} 2$ and $\mathrm{Ki}-67$ staining. Lower graphs, the expression of the indicated markers in each group was quantified, ${ }^{*} \mathrm{p}<0.05,{ }^{* *} \mathrm{P}<0.01$ indicates a significant difference vs. the vehicle. LicA, licochalcone. HK2, hexokinase 2.

(27-30). After LicA treatment, the expression of HK2 was substantially decreased, and both glucose absorption and lactate secretion in gastric cancer cells were significantly inhibited. Meanwhile, the western blotting results demonstrated that, except HK2, other key enzymes involved in glycolysis regulation had no obvious change. Additionally, Akt exogenous overexpression, which led to the recovery of HK2 expression, significantly rescued glycolysis suppression by LicA, suggesting that HK2 plays an important role in LicA-mediated suppression of tumor glycolysis.
The regulation of HK2 expression in tumor cells is complex. Like other glycolytic enzymes, its expression is primarily mediated by altered oncogenic pathways, such as PI3K-Akt, NF- $\mathrm{B}$, c-myc, HIF-1 $\alpha$ and p53 (31). After LicA treatment, the signaling pathways in gastric cancer cells including Akt, ERK and $\mathrm{NF}-\kappa \mathrm{B}$ were blocked. Further investigation clarified that HK2 downregulation was mainly attributed to the effect of LicA on the Akt signaling pathway. Except LY294002, other selective signaling pathway inhibitors had no effect on HK2 expression. Beyond that, HK2 reduction caused by LicA was dramatically 
attenuated after Akt overexpression, further confirming that the Akt signaling pathway is involved in the regulation of HK2 by LicA. In other cancers, such as colorectal (32), non-small cell lung cancer $(33,34)$, pediatric osteosarcoma (35), HK2 expression was also found to be regulated by Akt activity. However, further investigation is needed to elaborate the detailed mechanism of how HK2 is mediated by Akt.

Numerous studies had reported that LicA induced apoptosis in cancer cells via various mechanisms. In our study, the results demonstrated that HK2 plays a pivotal role in LicA-induced cell apoptosis in gastric cancer cells. Except the involvement of tumor glycolysis regulation, HK2 interacts with VDAC-1 on the outer mitochondria membrane to maintain membrane integrity under stressed condition and prevents cancer cells from apoptosis (36). With the decrease of HK2, gastric cancer cells underwent induced apoptosis by LicA, as evidenced by the increase in cleaved caspase-3 and PARP and the results of Annexin V-PI double staining. LicA-induced apoptosis was significantly declined after exogenous introduction of $\mathrm{HK} 2$, verifying that the decrease of HK2 was an important attributor to the apoptosis induction by LicA. Recent studies have reported that HK2 overexpression is closely correlated with chemotherapy resistance in various cancers, such as in epithelial ovarian, prostate and breast cancer (37-39). Given the effect of LicA on HK2, we speculate that LicA may have the potential to promote the efficacy of other chemotherapies in combinational usage.

Taken together, to the best of our knowledge, the present study is the first to report the effect of LicA on tumor glycolysis. We found that the reduction in HK2 was an important underlying mechanism for LicA to display its effects on glycolysis suppression and apoptosis induction. Moreover, we also disclosed that the decrease in HK2 caused by LicA was mainly attributed to the inhibition of the Akt signaling pathway. Our studies provide a preclinical rationale for LicA, or its derivatives to be administered for gastric cancer therapy.

\section{Acknowledgements}

The present study was supported by the Foundation of the Priority Academic Program Development of Jiangsu Higher Education Institutions (PAPD), the Jiangsu Provincial Special Program of Medical Science (BL2014100), the Jiangsu Hospital of TCM (Y14074) and the National Natural Science Foundation of China (nos. 81202954 and 81473605).

\section{References}

1. Lee $\mathrm{N}$ and Kim D: Cancer Metabolism: Fueling more than just growth. Mol Cells 39: 847-854, 2016.

2. Hanahan D and Weinberg RA: Hallmarks of cancer: The next generation. Cell 144: 646-674, 2011.

3. Doherty JR and Cleveland JL: Targeting lactate metabolism for cancer therapeutics. J Clin Invest 123: 3685-3692, 2013.

4. Rho M, Kim J, Jee CD, Lee YM, Lee HE, Kim MA, Lee HS and Kim WH: Expression of type 2 hexokinase and mitochondriarelated genes in gastric carcinoma tissues and cell lines. Anticancer Res 27A: 251-258, 2007.

5. Palmieri D, Fitzgerald D, Shreeve SM, Hua E, Bronder JL, Weil RJ, Davis S, Stark AM, Merino MJ, Kurek R, et al: Analyses of resected human brain metastases of breast cancer reveal the association between up-regulation of hexokinase 2 and poor prognosis. Mol Cancer Res 7: 1438-1445, 2009.
6. Liu Y, Wu K, Shi L, Xiang F, Tao K and Wang G: Prognostic significance of the metabolic marker hexokinase-2 in various solid tumors: A meta-analysis. PLoS One 11: e0166230, 2016.

7. Zhang ZF, Feng XS, Chen H, Duan ZJ, Wang LX, Yang D, Liu PX, Zhang QP, Jin YL, Sun ZG, et al: Prognostic significance of synergistic hexokinase-2 and beta2-adrenergic receptor expression in human hepatocelluar carcinoma after curative resection. BMC Gastroenterol 16: 57, 2016.

8. Kwee SA, Hernandez B, Chan O and Wong L: Choline kinase alpha and hexokinase-2 protein expression in hepatocellular carcinoma: Association with survival. PLoS One 7: e46591, 2012.

9. Hamabe A, Yamamoto H, Konno M, Uemura M, Nishimura J, Hata T, Takemasa I, Mizushima T, Nishida N, Kawamoto K, et al: Combined evaluation of hexokinase 2 and phosphorylated pyruvate dehydrogenase-E1 $\alpha$ in invasive front lesions of colorectal tumors predicts cancer metabolism and patient prognosis. Cancer Sci 105: 1100-1108, 2014.

10. Yu X, Deng Q, Li W, Xiao L, Luo X, Liu X, Yang L, Peng S, Ding Z, Feng T, et al: Neoalbaconol induces cell death through necroptosis by regulating RIPK-dependent autocrine TNF $\alpha$ and ROS production. Oncotarget 6: 1995-2008, 2015.

11. Yu X, Li W, Deng Q, You S, Liu H, Peng S, Liu X, Lu J, Luo X, Yang L, et al: Neoalbaconol inhibits angiogenesis and tumor growth by suppressing EGFR-mediated VEGF production. Mol Carcinog 56: 1414-1426, 2017.

12. Lee KW, Bode AM and Dong Z: Molecular targets of phytochemicals for cancer prevention. Nat Rev Cancer 11:211-218, 2011.

13. Chen X, Liu Z, Meng R, Shi C and Guo N: Antioxidative and anticancer properties of Licochalcone A from licorice. J Ethnopharmacol 198: 331-337, 2017.

14. Hu J and Liu J: Licochalcone A attenuates kipopolysaccharideinduced acute kidney injury by inhibiting NF- $\kappa \mathrm{B}$ activation. Inflammation 39: 569-574, 2016.

15. Wang L, Yang R, Yuan B, Liu Y and Liu C: The antiviral and antimicrobial activities of licorice, a widely-used Chinese herb. Acta Pharm Sin B 5: 310-315, 2015.

16. Bortolotto LF, Barbosa FR, Silva G, Bitencourt TA, Beleboni RO, Baek SJ, Marins M and Fachin AL: Cytotoxicity of trans-chalcone and licochalcone A against breast cancer cells is due to apoptosis induction and cell cycle arrest. Biomed Pharmacother 85 . 425-433, 2017

17. Tang ZH, Chen X, Wang ZY, Chai K, Wang YF, Xu XH, Wang XW, $\mathrm{Lu} \mathrm{JH}$, Wang YT, Chen XP, et al: Induction of C/EBP homologous protein-mediated apoptosis and autophagy by licochalcone A in non-small cell lung cancer cells. Sci Rep 6: 26241, 2016.

18. Park MR, Kim SG, Cho IA, Oh D, Kang KR, Lee SY, Moon SM, Cho SS, Yoon G, Kim CS, et al: Licochalcone-A induces intrinsic and extrinsic apoptosis via ERK1/2 and p38 phosphorylation-mediated TRAIL expression in head and neck squamous carcinoma FaDu cells. Food Chem Toxicol 77: 34-43, 2015.

19. Tsai JP, Lee CH, Ying TH, Lin CL, Lin CL, Hsueh JT and Hsieh YH: Licochalcone A induces autophagy through $\mathrm{PI} 3 \mathrm{~K} / \mathrm{Akt} / \mathrm{mTOR}$ inactivation and autophagy suppression enhances Licochalcone A-induced apoptosis of human cervical cancer cells. Oncotarget 6: 28851-28866, 2015.

20. Huang HC, Tsai LL, Tsai JP, Hsieh SC, Yang SF, Hsueh JT and Hsieh YH: Licochalcone A inhibits the migration and invasion of human lung cancer cells via inactivation of the Akt signaling pathway with downregulation of MMP-1/-3 expression. Tumour Biol 35: 12139-12149, 2014.

21. Kim YH, Shin EK, Kim DH, Lee HH, Park JH and Kim JK: Antiangiogenic effect of licochalcone A. Biochem Pharmacol 80: 1152-1159, 2010.

22. Chen W, Zheng R, Baade PD, Zhang S, Zeng H, Bray F, Jemal A Yu XQ and He J: Cancer statistics in China, 2015. CA Cancer J Clin 66: 115-132, 2016.

23. Kinghorn AD, DE Blanco EJ, Lucas DM, Rakotondraibe HL, Orjala J, Soejarto DD, Oberlies NH, Pearce CJ, Wani MC, Stockwell BR, et al: Discovery of anticancer agents of diverse natural origin. Anticancer Res 36: 5623-5637, 2016.

24. Hao W, Yuan X, Yu L, Gao C, Sun X, Wang D and Zheng Q: Licochalcone A-induced human gastric cancer BGC-823 cells apoptosis by regulating ROS-mediated MAPKs and PI3K/AKT signaling pathways. Sci Rep 5: 10336, 2015.

25. Lee JW, Lee SM, Lee MS and Shin HC: Role of ${ }^{18}$ F-FDG PET/CT in the prediction of gastric cancer recurrence after curative surgical resection. Eur J Nucl Med Mol Imaging 39: 1425-1434, 2012.

26. Coupe NA, Karikios D, Chong S, Yap J, Ng W, Merrett N and Lin M: Metabolic information on staging FDG-PET-CT as a prognostic tool in the evaluation of 97 patients with gastric cancer. Ann Nucl Med 28: 128-135, 2014. 
27. Watanabe Y, Suefuji H, Hirose Y, Kaida H, Suzuki G, Uozumi J, Ogo E, Miura M, Takasu K, Miyazaki K, et al: ${ }^{18} \mathrm{~F}-\mathrm{FDG}$ uptake in primary gastric malignant lymphoma correlates with glucose transporter 1 expression and histologic malignant potential. Int J Hematol 97: 43-49, 2013.

28. Qiu MZ, Han B, Luo HY, Zhou ZW, Wang ZQ, Wang FH, $\mathrm{Li}$ YH and Xu RH: Expressions of hypoxia-inducible factor-1a and hexokinase-II in gastric adenocarcinoma: The impact on prognosis and correlation to clinicopathologic features. Tumour Biol 32: 159-166, 2011.

29. Yin L, Wang X, Luo C, Liu H, Zhang L, Zhang H and Zhang Y: The value of expression of M2-PK and VEGF in patients with advanced gastric cancer. Cell Biochem Biophys 67: 1033-1039, 2013.

30. Lim JY, Yoon SO, Seol SY, Hong SW, Kim JW, Choi SH and Cho JY: Overexpression of the M2 isoform of pyruvate kinase is an adverse prognostic factor for signet ring cell gastric cancer. World J Gastroenterol 18: 4037-4043, 2012.

31. Mathupala SP, Ko YH and Pedersen PL: Hexokinase II: Cancer's double-edged sword acting as both facilitator and gatekeeper of malignancy when bound to mitochondria. Oncogene 25 . 4777-4786, 2006

32. Chen GQ, Tang CF, Shi XK, Lin CY, Fatima S, Pan XH, Yang DJ, Zhang G, Lu AP, Lin SH, et al: Halofuginone inhibits colorectal cancer growth through suppression of Akt/mTORC1 signaling and glucose metabolism. Oncotarget 6: 24148-24162, 2015.

33. Li W, Ma X, Li N, Liu H, Dong Q, Zhang J, Yang C, Liu Y, Liang Q, Zhang S, et al: Resveratrol inhibits Hexokinases II mediated glycolysis in non-small cell lung cancer via targeting Akt signaling pathway. Exp Cell Res 349: 320-327, 2016.
34. Li W, Gao F, Ma X, Wang R, Dong X and Wang W: Deguelin inhibits non-small cell lung cancer via down-regulating Hexokinases II-mediated glycolysis. Oncotarget 8: 32586-32599, 2017.

35. Zhuo B, Li Y, Li Z, Qin H, Sun Q, Zhang F, Shen Y, Shi Y and Wang R: PI3K/Akt signaling mediated Hexokinase-2 expression inhibits cell apoptosis and promotes tumor growth in pediatric osteosarcoma. Biochem Biophys Res Commun 464: 401-406, 2015.

36. Zhou Y, Lu N, Qiao C, Ni T, Li Z, Yu B, Guo Q and Wei L: FV-429 induces apoptosis and inhibits glycolysis by inhibiting Akt-mediated phosphorylation of hexokinase II in MDA-MB-231 cells. Mol Carcinog 55: 1317-1328, 2016.

37. Suh DH, Kim MA, Kim H, Kim MK, Kim HS, Chung HH, Kim YB and Song YS: Association of overexpression of hexokinase II with chemoresistance in epithelial ovarian cancer. Clin Exp Med 14: 345-353, 2014.

38. Wang L, Wang J, Xiong H, Wu F, Lan T, Zhang Y, Guo X, Wang H, Saleem M, Jiang C, et al: Co-targeting hexokinase 2-mediated Warburg effect and ULK1-dependent autophagy suppresses tumor growth of PTEN- and TP53-deficiency-driven castrationresistant prostate cancer. EBioMedicine 7: 50-61, 2016.

39. Wang J, Duan Z, Nugent Z, Zou JX, Borowsky AD, Zhang Y, Tepper CG, Li JJ, Fiehn O, Xu J, et al: Reprogramming metabolism by histone methyltransferase NSD2 drives endocrine resistance via coordinated activation of pentose phosphate pathway enzymes. Cancer Lett 378: 69-79, 2016. 